\title{
DIGITAL EXPANSIONS WITH NEGATIVE REAL BASES
}

\author{
WOLFGANG STEINER
}

\begin{abstract}
Similarly to Parry's characterization of $\beta$-expansions of real numbers in real bases $\beta>1$, Ito and Sadahiro characterized digital expansions in negative bases, by the expansions of the endpoints of the fundamental interval. Parry also described the possible expansions of 1 in base $\beta>1$. In the same vein, we characterize the sequences that occur as $(-\beta)$-expansion of $\frac{-\beta}{\beta+1}$ for some $\beta>1$. These sequences also describe the itineraries of 1 by linear mod one transformations with negative slope.
\end{abstract}

\section{INTRODUCTION}

Digital expansions in real bases $\beta>1$ were introduced by Rényi [Rén57]: The (greedy) $\beta$-expansion of a real number $x \in[0,1)$ is

$$
x=\frac{\varepsilon_{1}(x)}{\beta}+\frac{\varepsilon_{2}(x)}{\beta^{2}}+\cdots \quad \text { with } \quad \varepsilon_{n}(x)=\left\lfloor\beta T_{\beta}^{n-1}(x)\right\rfloor,
$$

where $\lfloor\cdot\rfloor$ denotes the floor function and $T_{\beta}$ is the $\beta$-transformation

$$
T_{\beta}:[0,1) \rightarrow[0,1), \quad x \mapsto \beta x-\lfloor\beta x\rfloor .
$$

Rényi suggested representing arbitrary $x \in \mathbb{R}$ by

$$
x=\lfloor x\rfloor+\frac{\varepsilon_{1}(\lfloor x\rfloor)}{\beta}+\frac{\varepsilon_{2}(\lfloor x\rfloor)}{\beta^{2}}+\cdots,
$$

whereas nowadays it is more usual (for $x \geq 0$ ) to multiply the $\beta$-expansion of $x \beta^{-k}$ by $\beta^{k}$, with $k$ an arbitrary integer satisfying $x \beta^{-k} \in[0,1)$. Anyway, the possible expansions can be described by those of $x \in[0,1)$. A sequence $b_{1} b_{2} \cdots$ is called $\beta$-admissible if and only if it is (the digit sequence of) the $\beta$-expansion of a number $x \in[0,1)$, i.e., $b_{n}=\varepsilon_{n}(x)$ for all $n \geq 1$. Parry [Par60] showed that an integer sequence $b_{1} b_{2} \cdots$ is $\beta$-admissible if and only if

$$
00 \cdots \leq_{\text {lex }} b_{k} b_{k+1} \cdots<_{\text {lex }} a_{1} a_{2} \cdots \quad \text { for all } k \geq 1,
$$

where $<_{\text {lex }}$ denotes the lexicographic order and $a_{1} a_{2} \ldots$ is the (quasi-greedy) $\beta$-expansion of 1, i.e., $a_{n}=\lim _{x \rightarrow 1-} \varepsilon_{n}(x)$. Moreover, a sequence of integers $a_{1} a_{2} \ldots$ is the (quasigreedy) $\beta$-expansion of 1 for some $\beta>1$ if and only if

$$
00 \cdots<_{\operatorname{lex}} a_{k} a_{k+1} \cdots \leq_{\operatorname{lex}} a_{1} a_{2} \cdots \quad \text { for all } k \geq 2 .
$$

(These results are stated in a slightly different way in Par60.)

Part of this research was conducted while the author was visiting academic at the Department of Computing of the Macquarie University, Sydney. 
Following [Rén57] and [Par60, a lot of papers were dedicated to the study of $\beta$-expansions and $\beta$-transformations, but surprisingly little attention was given to digital expansions in negative bases. This changed only in recent years, after Ito and Sadahiro [IS09] considered $(-\beta)$-expansions, $\beta>1$, defined for $x \in\left[\frac{-\beta}{\beta+1}, \frac{1}{\beta+1}\right)$ by

$$
x=\frac{\varepsilon_{1}(x)}{-\beta}+\frac{\varepsilon_{2}(x)}{(-\beta)^{2}}+\cdots \quad \text { with } \quad \varepsilon_{n}(x)=\left\lfloor\frac{\beta}{\beta+1}-\beta T_{-\beta}^{n-1}(x)\right\rfloor,
$$

where the $(-\beta)$-transformation is defined by

$$
T_{-\beta}:\left[\frac{-\beta}{\beta+1}, \frac{1}{\beta+1}\right) \rightarrow\left[\frac{-\beta}{\beta+1}, \frac{1}{\beta+1}\right), \quad x \mapsto-\beta x-\left\lfloor\frac{\beta}{\beta+1}-\beta x\right\rfloor .
$$

A sequence $b_{1} b_{2} \cdots$ is $(-\beta)$-admissible if and only if it is the $(-\beta)$-expansion of some $x \in\left[\frac{-\beta}{\beta+1}, \frac{1}{\beta+1}\right)$, i.e., $b_{n}=\varepsilon_{n}(x)$ for all $n \geq 1$. Since the map $x \mapsto-\beta x$ is order-reversing, the $(-\beta)$-admissible sequences are characterized using the alternating lexicographic order. By [IS09], a sequence $b_{1} b_{2} \cdots$ is $(-\beta)$-admissible if and only if

$$
a_{1} a_{2} \cdots \geq_{\text {alt }} b_{k} b_{k+1} \cdots>_{\text {alt }} 0 a_{1} a_{2} \cdots \quad \text { for all } k \geq 1,
$$

where $a_{1} a_{2} \cdots$ is the $(-\beta)$-expansion of the left endpoint $\frac{-\beta}{\beta+1}$, i.e., $a_{n}=\varepsilon_{n}\left(\frac{-\beta}{\beta+1}\right)$, which is supposed not to be periodic with odd period length. If $a_{1} a_{2} \cdots=\overline{a_{1} a_{2} \cdots a_{2 \ell+1}}$ for some $\ell \geq 0$, and $\ell$ is minimal with this property, then the condition (1.2) is replaced by

$$
a_{1} a_{2} \cdots \geq_{\text {alt }} b_{k} b_{k+1} \cdots>_{\text {alt }} \overline{0 a_{1} \cdots a_{2 \ell}\left(a_{2 \ell+1}-1\right)} \text { for all } k \geq 1 .
$$

Recall that the alternating lexicographic order is defined on sequences $x_{1} x_{2} \cdots, y_{1} y_{2} \cdots$ with $x_{1} \cdots x_{k-1}=y_{1} \cdots y_{k-1}$ and $x_{k} \neq y_{k}$ by

$$
x_{1} x_{2} \cdots<_{\text {alt }} y_{1} y_{2} \cdots \quad \text { if and only if } \begin{cases}x_{k}<y_{k} & \text { when } k \text { is odd, } \\ y_{k}<x_{k} & \text { when } k \text { is even. }\end{cases}
$$

The main result of this paper is a characterization of the sequences $a_{1} a_{2} \cdots$ that are the $(-\beta)$-expansion of $\frac{-\beta}{\beta+1}$ for some $\beta>1$. This turns out to be more complicated than the corresponding problem for $\beta$-expansions, and we will see that several proofs cannot be directly carried over from positive to negative bases. From (1.2) and (1.3), one deduces that

$$
a_{k} a_{k+1} \cdots \leq_{\text {alt }} a_{1} a_{2} \cdots \text { for all } k \geq 2 .
$$

The proof of Proposition 3.5 in [LS] (see also Theorem 3 below) shows that

$$
a_{1} a_{2} \cdots>_{\text {alt }} u_{1} u_{2} \cdots=100111001001001110011 \cdots
$$

where $u_{1} u_{2} \cdots$ is the sequence starting with $\varphi^{n}(1)$ for all $n \geq 0$, with $\varphi$ being the morphism of words on the alphabet $\{0,1\}$ defined by $\varphi(1)=100, \varphi(0)=1$. (See the remarks following Theorem 3 and note that the alphabet is shifted by 1 in [LS].) Our first result states that a sequence satisfying (1.4) and (1.5) is "almost" the $(-\beta)$-expansion of $\frac{-\beta}{\beta+1}$ for some $\beta>1$. 
Theorem 1. Let $a_{1} a_{2} \cdots$ be a sequence of non-negative integers satisfying (1.4) and (1.5). Then there exists a unique $\beta>1$ such that

$$
\sum_{j=1}^{\infty} \frac{a_{j}}{(-\beta)^{j}}=\frac{-\beta}{\beta+1} \quad \text { and } \quad \sum_{j=1}^{\infty} \frac{a_{k+j}}{(-\beta)^{j}} \in\left[\frac{-\beta}{\beta+1}, \frac{1}{\beta+1}\right] \quad \text { for all } k \geq 1 \text {. }
$$

For a $(-\beta)$-expansion of $\frac{-\beta}{\beta+1}$, we have to exclude the possibility that $\sum_{j=1}^{\infty} \frac{a_{k+j}}{(-\beta)^{j}}=\frac{1}{\beta+1}$ for some $k \geq 1$. If $\overline{a_{1} \cdots a_{k}}>$ alt $u_{1} u_{2} \cdots$, then out of $\left\{a_{1} \cdots a_{k}, a_{1} \cdots a_{k-1}\left(a_{k}-1\right) 0\right\}{ }^{\omega}$, which is the set of infinite sequences composed of blocks $a_{1} \cdots a_{k}$ and $a_{1} \cdots a_{k-1}\left(a_{k}-1\right) 0$, only the periodic sequence $\overline{a_{1} \cdots a_{k}}$ is possibly the $(-\beta)$-expansion of $\frac{-\beta}{\beta+1}$ for some $\beta>1$, see Section 4. This implies that

$$
\begin{aligned}
& a_{1} a_{2} \cdots \notin\left\{a_{1} \cdots a_{k}, a_{1} \cdots a_{k-1}\left(a_{k}-1\right) 0\right\}^{\omega} \backslash\left\{\overline{a_{1} \cdots a_{k}}\right\} \\
& \text { for all } k \geq 1 \text { with } \overline{a_{1} \cdots a_{k}} \succ u_{1} u_{2} \cdots, \\
& a_{1} a_{2} \cdots \notin\left\{a_{1} \cdots a_{k} 0, a_{1} \cdots a_{k-1}\left(a_{k}+1\right)\right\}^{\omega} \\
& \\
& \text { for all } k \geq 1 \text { with } \overline{a_{1} \cdots a_{k-1}\left(a_{k}+1\right)} \succ u_{1} u_{2} \cdots .
\end{aligned}
$$

The main result states that there are no other conditions on $a_{1} a_{2} \cdots$.

Theorem 2. A sequence of non-negative integers $a_{1} a_{2} \cdots$ is the $(-\beta)$-expansion of $\frac{-\beta}{\beta+1}$ for some (unique) $\beta>1$ if and only if it satisfies (1.4), (1.5), (1.7), and (1.8).

It is easy to see that the natural order of bases $\beta>1$ is reflected by the lexicographical order of the (quasi-greedy) $\beta$-expansions of 1 [Par60]. For negative bases, a similar relation with the alternating lexicographic order holds, although it is a bit harder to prove.

Theorem 3. Let $a_{1} a_{2} \cdots$ be the $(-\beta)$-expansion of $\frac{-\beta}{\beta+1}$ and $a_{1}^{\prime} a_{2}^{\prime} \cdots$ be the $\left(-\beta^{\prime}\right)$-expansion of $\frac{-\beta^{\prime}}{\beta^{\prime}+1}$, with $\beta, \beta^{\prime}>1$. Then $\beta<\beta^{\prime}$ if and only if $a_{1} a_{2} \cdots<_{\text {alt }} a_{1}^{\prime} a_{2}^{\prime} \cdots$.

It is often convenient to study a slightly different $(-\beta)$-transformation,

$$
\widetilde{T}_{-\beta}:(0,1] \rightarrow(0,1], \quad x \mapsto-\beta x+\lfloor\beta x\rfloor+1 .
$$

As already noted in [LS], the transformations $T_{-\beta}$ and $\widetilde{T}_{-\beta}$ are conjugate via the involution $\phi(x)=\frac{1}{\beta+1}-x$, i.e.,

$$
T_{-\beta} \circ \phi(x)=\phi \circ \widetilde{T}_{-\beta}(x) \text { for all } x \in(0,1] .
$$

Setting $\tilde{\varepsilon}_{n}(x)=\left\lfloor\beta \widetilde{T}_{-\beta}^{n-1}(x)\right\rfloor$ for $x \in(0,1]$, we have $x=-\sum_{n=1}^{\infty} \frac{\tilde{\varepsilon}_{n}(x)+1}{(-\beta)^{n}}=\frac{1}{\beta+1}-\sum_{n=1}^{\infty} \frac{\tilde{\varepsilon}_{n}(x)}{(-\beta)^{n}}$, and $\tilde{\varepsilon}_{n}(x)=\varepsilon_{n}(\phi(x))$. Note that $\widetilde{T}_{-\beta}(x)=-\beta x-\lfloor-\beta x\rfloor$ except for finitely many points, hence $\widetilde{T}_{-\beta}$ is a natural generalization of the beta-transformation. The map $\widetilde{T}_{-\beta}$ was studied e.g. by Góra Gór07, where it corresponds to the case $E=[1,1, \ldots, 1]$, and in [LS]. The following corollary is an immediate consequence of Theorems 1 and 2. 
Corollary 1. Let $a_{1} a_{2}$ ․ be a sequence of non-negative integers satisfying (1.4) and (1.5). Then there exists a unique $\beta>1$ such that

$$
-\sum_{j=1}^{\infty} \frac{a_{j}+1}{(-\beta)^{j}}=1 \quad \text { and } \quad-\sum_{j=1}^{\infty} \frac{a_{k+j}+1}{(-\beta)^{j}} \in[0,1] \quad \text { for all } k \geq 1 .
$$

Moreover, $\sum_{j=1}^{\infty} \frac{a_{k+j}+1}{(-\beta)^{j}} \neq 0$ for all $k \geq 1$ if and only if (1.7) and (1.8) hold.

With the notation of [Gór07, this means, for $E=[1,1, \ldots, 1]$, that $a_{1} a_{2} \cdots$ is the itinerary $\operatorname{It}_{\beta}(1)$ for some $\beta>1$ if and only if (1.4), (1.5), (1.7), and (1.8) hold. Note that Góra Gór07, Theorems 25 and 28] claims that already (1.4) is sufficient when $a_{1} \geq 2$, and he has a less explicit statement for $a_{1}=1$. However, his proof deals only with the first part of the theorem, i.e., that there exists a unique $\beta>1$ satisfying (1.9). To see that this is not sufficient, consider the sequences $a_{1} a_{2} \cdots \in\{2,10\}^{\omega}$. They all satisfy (1.9) with $\beta=2$, and there are uncountably many of them satisfying (1.4) and $a_{1}=2$. All these uncountably many sequences would have to be equal to $\mathrm{It}_{2}(1)$ by Gór07. Theorem 25], which is of course not true. (See also [DMP11].) Moreover, Góra's proof of the existence of a unique $\beta>1$ satisfying (1.9) is incorrect when $\beta$ is small, see Remark 1 .

\section{Proof of Theorem 3}

Let $\beta>1$. For a sequence of digits $b_{1} \cdots b_{n}$, set

$$
I_{b_{1} \cdots b_{n}}=\left\{x \in\left[\frac{-\beta}{\beta+1}, \frac{1}{\beta+1}\right): \varepsilon_{1}(x) \cdots \varepsilon_{n}(x)=b_{1} \cdots b_{n}\right\},
$$

with $\varepsilon_{j}(x)$ as in (1.1). Let $L_{\beta, n}$ be the number of different sequences $b_{1} \cdots b_{n}$ such that $I_{b_{1} \cdots b_{n}} \neq \emptyset$, and let $L_{\beta, n}^{\prime}$ be the number of different sequences $b_{1} \cdots b_{n}$ such that $I_{b_{1} \cdots b_{n}}$ is an interval of positive length. (The latter is called the lap number of $T_{-\beta}^{n}$.)

Lemma 1. For any $\beta>1$, we have that $\lim _{n \rightarrow \infty} \frac{1}{n} \log L_{\beta, n}=\lim _{n \rightarrow \infty} \frac{1}{n} \log L_{\beta, n}^{\prime}=\log \beta$.

Proof. It is well known that the entropy of $T_{-\beta}$, which is a piecewise linear map of constant slope $-\beta$, is $\log \beta$. The lemma can be derived from this fact, see [FL11], but we prefer giving a short elementary proof, following Faller [Fal08, Proposition 3.6]. As $\left|\frac{d}{d x} T_{-\beta}^{n}(x)\right|=\beta^{n}$ at all points of continuity of $T_{-\beta}^{n}$, the length of any interval $I_{b_{1} \cdots b_{n}}$ is at most $\beta^{-n}$. Since the intervals $I_{b_{1} \cdots b_{n}}$ form a partition of an interval of length 1 , we obtain that $L_{\beta, n} \geq L_{\beta, n}^{\prime} \geq \beta^{n}$.

To get an upper bound for $L_{\beta, n}^{\prime}$, let $m$ be the smallest positive integer such that $\beta^{m}>2$, and let $\delta$ be the minimal positive length of an interval $I_{b_{1} \cdots b_{m}}$. Consider an interval $I_{b_{1} \cdots b_{n}}$, $n>m$, such that $b_{1} \cdots b_{n}$ is neither the minimal nor the maximal sequence (with respect to the alternating lexicographic order) starting with $b_{1} \cdots b_{n-m}$ and satisfying $I_{b_{1} \cdots b_{n}} \neq \emptyset$. Then each prolongation $b_{1} b_{2} \cdots$ satisfies the inequalities in (1.2) and (1.3), respectively, for $1 \leq k \leq n-m$. Therefore, $b_{1} b_{2} \cdots$ is $(-\beta)$-admissible if and only if $b_{n-m+1} b_{n-m+2} \cdots$ is $(-\beta)$-admissible. This implies that $T_{-\beta}^{n-m}\left(I_{b_{1} \cdots b_{n}}\right)=I_{b_{n-m+1} \cdots b_{m}}$, and the length of $I_{b_{1} \cdots b_{n}}$ is $\beta^{m-n}$ times the length of $I_{b_{n-m+1} \cdots b_{m}}$, thus at least $\beta^{m-n} \delta$ when the length is positive. There are at least $L_{\beta, n}^{\prime}-2 L_{\beta, n-m}^{\prime}$ sequences $b_{1} \cdots b_{n}$ such that $I_{b_{1} \cdots b_{n}}$ has positive length 
and $b_{1} \cdots b_{n}$ is neither the minimal nor the maximal sequence starting with $b_{1} \cdots b_{n-m}$ and satisfying $I_{b_{1} \cdots b_{n}} \neq \emptyset$. This yields that $\left(L_{\beta, n}^{\prime}-2 L_{\beta, n-m}^{\prime}\right) \beta^{m-n} \delta \leq 1$ for all $n>m$, thus

$$
\begin{aligned}
& L_{\beta, n}^{\prime} \leq \frac{\beta^{n-m}}{\delta}+2 L_{\beta, n-m}^{\prime} \leq \frac{\beta^{n-m}}{\delta}+\frac{2 \beta^{n-2 m}}{\delta}+4 L_{\beta, n-2 m}^{\prime} \leq \cdots \\
& \leq \frac{\beta^{n-m}}{\delta} \sum_{j=0}^{\lceil n / m\rceil-2}\left(\frac{2}{\beta^{m}}\right)^{j}+2^{\lceil n / m\rceil-1} L_{\beta, n-\lceil n / m\rceil m+m}^{\prime}<\frac{\beta^{n}}{\delta} \frac{1}{\beta^{m}-2}+\beta^{n} L_{\beta, m}^{\prime} \leq \frac{\beta^{n}}{\delta} \frac{\beta^{m}-1}{\beta^{m}-2} .
\end{aligned}
$$

This shows that $\lim _{n \rightarrow \infty} \frac{1}{n} \log L_{\beta, n}^{\prime}=\beta$.

An interval $I_{b_{1} \cdots b_{n}}$ consists only of one point if and only if $I_{b_{1} \cdots b_{k}}=\left\{\frac{-\beta}{\beta+1}\right\}$ and $b_{k+1} \cdots b_{n}=$ $a_{1} \cdots a_{n-k}$ for some $k \leq n$. (This can happen only in case that $a_{1} a_{2} \cdots$ is periodic with odd period length.) Therefore, we can estimate $L_{\beta, n}-L_{\beta, n}^{\prime} \leq L_{\beta, 0}^{\prime}+L_{\beta, 1}^{\prime}+\cdots+L_{\beta, n}^{\prime} \leq C \beta^{n}$ for some constant $C>0$, thus $\lim _{n \rightarrow \infty} \frac{1}{n} \log L_{\beta, n}=\lim _{n \rightarrow \infty} \frac{1}{n} \log L_{\beta, n}^{\prime}$.

For the proof of Theorem 3 , let $a_{1} a_{2} \cdots$ be the $(-\beta)$-expansion of $\frac{-\beta}{\beta+1}$ and $a_{1}^{\prime} a_{2}^{\prime} \cdots$ be the $\left(-\beta^{\prime}\right)$-expansion of $\frac{-\beta^{\prime}}{\beta^{\prime}+1}, \beta, \beta^{\prime}>1$. If $\beta=\beta^{\prime}$, then we clearly have that $a_{1} a_{2} \cdots=a_{1}^{\prime} a_{2}^{\prime} \cdots$. If $a_{1} a_{2} \cdots=a_{1}^{\prime} a_{2}^{\prime} \cdots$, then the $(-\beta)$-admissible sequences are equal to the $\left(-\beta^{\prime}\right)$-admissible sequences, thus $L_{\beta, n}=L_{\beta^{\prime}, n}$ for all $n \geq 1$, and $\beta=\beta^{\prime}$ by Lemma 11. Therefore, the equations $\beta=\beta^{\prime}$ and $a_{1} a_{2} \cdots=a_{1}^{\prime} a_{2}^{\prime} \cdots$ are equivalent. Hence, it suffices to show that $a_{1} a_{2} \cdots<_{\text {alt }} a_{1}^{\prime} a_{2}^{\prime} \cdots$ implies that $\beta<\beta^{\prime}$, as the other direction follows by contraposition.

Assume that $a_{1} a_{2} \cdots<_{\text {alt }} a_{1}^{\prime} a_{2}^{\prime} \cdots$, and let $b_{1} b_{2} \cdots$ be a $(-\beta)$-admissible sequence. By (1.2) and (1.3) respectively, we have that

$$
b_{k} b_{k+1} \cdots \leq_{\text {alt }} a_{1} a_{2} \cdots<_{\text {alt }} a_{1}^{\prime} a_{2}^{\prime} \cdots .
$$

Furthermore, as $\overline{0 a_{1} \cdots a_{2 \ell}\left(a_{2 \ell+1}-1\right)}>$ alt $0 a_{1} a_{2} \cdots$ for all $\ell \geq 0$, we obtain that

$$
b_{k} b_{k+1} \cdots>_{\text {alt }} 0 a_{1} a_{2} \cdots>_{\text {alt }} 0 a_{1}^{\prime} a_{2}^{\prime} \cdots .
$$

If $a_{1}^{\prime} a_{2}^{\prime} \cdots$ is not periodic with odd period length, then (2.1) and (2.2) show that $b_{1} b_{2} \cdots$ is $\left(-\beta^{\prime}\right)$-admissible, thus $L_{\beta, n} \leq L_{\beta^{\prime}, n}$ for all $n \geq 1$, and $\beta \leq \beta^{\prime}$ by Lemma 1. Since $a_{1} a_{2} \cdots \neq a_{1}^{\prime} a_{2}^{\prime} \cdots$, this yields that $\beta<\beta^{\prime}$. In case $a_{1}^{\prime} a_{2}^{\prime} \cdots=\overline{a_{1}^{\prime} \cdots a_{2 \ell^{\prime}+1}^{\prime}}$, we show that

$$
a_{1} a_{2} \cdots \leq_{\text {alt }} \overline{a_{1}^{\prime} \cdots a_{2 \ell^{\prime}}^{\prime}\left(a_{2 \ell^{\prime}+1}^{\prime}-1\right) 0}
$$

This is clearly true when $a_{1} \cdots a_{2 \ell^{\prime}+1}<_{\text {alt }} a_{1}^{\prime} \cdots a_{2 \ell^{\prime}}^{\prime}\left(a_{2 \ell^{\prime}+1}^{\prime}-1\right)$. If $a_{1} \cdots a_{2 \ell^{\prime}+1}=a_{1}^{\prime} \cdots a_{2 \ell^{\prime}+1}^{\prime}$, then $a_{2 \ell^{\prime}+2} a_{2 \ell^{\prime}+3} \cdots>_{\text {alt }} a_{2 \ell^{\prime}+2}^{\prime} a_{2 \ell^{\prime}+3}^{\prime} \cdots=a_{1}^{\prime} a_{2}^{\prime} \cdots>_{\text {alt }} a_{1} a_{2} \cdots$, contradicting (1.4). It remains to consider the case that $a_{1} \cdots a_{2 \ell^{\prime}+1}=a_{1}^{\prime} \cdots a_{2 \ell^{\prime}}^{\prime}\left(a_{2 \ell^{\prime}+1}^{\prime}-1\right)$. If $a_{2 \ell^{\prime}+1}>0$, then (2.3) holds, otherwise $a_{1} \cdots a_{2 \ell^{\prime}+2}=a_{1}^{\prime} \cdots a_{2 \ell^{\prime}}^{\prime}\left(a_{2 \ell^{\prime}+1}^{\prime}-1\right) 0$. In the latter case, (1.4) implies that $a_{2 \ell^{\prime}+3} \cdots a_{4 \ell^{\prime}+4} \leq_{\text {alt }} a_{1} \cdots a_{2 \ell^{\prime}+2}=a_{1}^{\prime} \cdots a_{2 \ell^{\prime}}^{\prime}\left(a_{2 \ell^{\prime}+1}^{\prime}-1\right) 0$, and we obtain inductively that (2.3) holds. Now, (2.1), (2.2), and (2.3) show that $b_{1} b_{2} \cdots$ is $\left(-\beta^{\prime}\right)$-admissible, which yields as above that $\beta<\beta^{\prime}$. 


\section{Proof of Theorem 1}

Let $a_{1} a_{2} \cdots$ be a sequence of non-negative integers satisfying (1.4) and (1.5). We show that there exists a unique $\beta>1$ satisfying (1.9), which is equivalent to (1.6) $)$. For $n \geq 1$, set

$$
\begin{gathered}
P_{n}(x)=(-x)^{n}+\sum_{j=1}^{n}\left(a_{j}+1\right)(-x)^{n-j}, \\
J_{n}=\left\{x>1 \mid P_{j}(x) \in[0,1] \text { for all } 1 \leq j \leq n\right\} .
\end{gathered}
$$

Then $J_{1} \supseteq J_{2} \supseteq J_{3} \supseteq \cdots$, and $J_{n}$ is compact if and only if inf $J_{n} \neq 1$.

First note that, for $\beta>1$, (1.9) is equivalent to $\beta \in \bigcap_{n \geq 1} J_{n}$. Indeed, if (1.9) holds, then $P_{n}(\beta)=-\sum_{j=1}^{\infty} \frac{a_{n+j}+1}{(-\beta)^{j}} \in[0,1]$ for all $n \geq 1$. On the other hand, if $P_{n}(\beta) \in[0,1]$ for all $n \geq 1$, then $\left|1+\sum_{j=1}^{\infty} \frac{a_{j}+1}{(-\beta)^{j}}\right|=\lim _{n \rightarrow \infty} \frac{P_{n}(\beta)}{(-\beta)^{n}}=0$, thus (1.9) holds.

Inductively for $n \geq 1$, we show the following statements, where we use the abbreviations $v_{[j, k]}$ for $v_{j} v_{j+1} \cdots v_{k}$ and $v_{[j, k)}$ for $v_{j} v_{j+1} \cdots v_{k-1}$ :

(1) $J_{n}$ is a non-empty interval, with $\inf J_{n}=1$ if and only if $a_{[1, n]}=u_{[1, n]}$. If $P_{n}(\beta)=P_{n}\left(\beta^{\prime}\right) \in\{0,1\}$ with $\beta, \beta^{\prime} \in J_{n}$, then $\beta=\beta^{\prime}$.

(2) If $n$ is even, $a_{[1, n-2 m+1]}=u_{[1, n-2 m+1]}$ or $a_{[n-2 m+2, n]} \neq a_{[1,2 m)}$ for all $1 \leq m \leq n / 2$, and $a_{[1, n]} \neq u_{[1, n]}$, then $P_{n}\left(\min J_{n}\right)=0$.

If $n$ is odd and $a_{[n-2 m+2, n]} \neq a_{[1,2 m)}$ for all $1 \leq m \leq n / 2$, then $P_{n}\left(\max J_{n}\right)=0$.

(3) If $n$ is even, $a_{[1, n-2 m+1]} \neq u_{[1, n-2 m+1]}$ and $a_{[n-2 m+2, n]}=a_{[1,2 m)}$ for some $1 \leq m \leq n / 2$, and $m$ is maximal with this property, then $P_{n}\left(\min J_{n}\right)=P_{2 m-1}\left(\min J_{n}\right)$.

If $n$ is odd, $a_{[n-2 m+2, n]}=a_{[1,2 m)}$ for some $1 \leq m \leq n / 2$, and $m$ is maximal with this property, then $P_{n}\left(\max J_{n}\right)=P_{2 m-1}\left(\max J_{n}\right)$.

(4) If $n$ is even and $a_{[n-2 m+1, n]} \neq a_{[1,2 m]}$ for all $1 \leq m<n / 2$, then $P_{n}\left(\max J_{n}\right)=1$.

If $n$ is odd, $a_{[1, n-2 m]}=u_{[1, n-2 m]}$ or $a_{[n-2 m+1, n]} \neq a_{[1,2 m]}$ for all $1 \leq m<n / 2$, and $a_{[1, n]} \neq u_{[1, n]}$, then $P_{n}\left(\min J_{n}\right)=1$.

(5) If $n$ is even, $a_{[n-2 m+1, n]}=a_{[1,2 m]}$ for some $1 \leq m<n / 2$, and $m$ is maximal with this property, then $P_{n}\left(\max J_{n}\right)=P_{2 m}\left(\max J_{n}\right)$.

If $n$ is odd, $a_{[1, n-2 m]} \neq u_{[1, n-2 m]}$ and $a_{[n-2 m+1, n]}=a_{[1,2 m]}$ for some $1 \leq m<n / 2$, and $m$ is maximal with this property, then $P_{n}\left(\min J_{n}\right)=P_{2 m}\left(\min J_{n}\right)$.

We have that $P_{1}(x)=a_{1}+1-x$, and $a_{1} \geq 1$ by (1.5). If $a_{1} \geq 2$, then $J_{1}=\left[a_{1}, a_{1}+1\right]$, $P_{1}\left(a_{1}\right)=1$ and $P_{1}\left(a_{1}+1\right)=0$; if $a_{1}=1$, then $J_{1}=(1,2]$ and $P_{1}(2)=0$. Therefore, the statements hold for $n=1$. Assume that they hold for $n-1$, and set

$$
B=\left\{b \in\left\{0,1, \ldots, a_{1}\right\}: b+1-x P_{n-1}(x) \in[0,1] \text { for some } x \in J_{n-1}\right\},
$$

i.e., $J_{n} \neq \emptyset$ if and only if $a_{n} \in B$.

Assume first that $a_{[1, n)} \neq u_{[1, n)}$, i.e., inf $J_{n-1}=\min J_{n-1}>1$, and that $n$ is even.

(i) If $a_{[n-2 m+1, n)} \neq a_{[1,2 m)}$ for all $1 \leq m<n / 2$, then $P_{n-1}\left(\max J_{n-1}\right)=0$, thus

$$
1-\left(\max J_{n-1}\right) P_{n-1}\left(\max J_{n-1}\right)=1 \text {. }
$$

This implies that $0 \in B$, and $P_{n}\left(\max J_{n}\right)=P_{n}\left(\max J_{n-1}\right)=1$ if $a_{n}=0$. Since the map $x \mapsto x P_{n-1}(x)$ is continuous and $J_{n-1}$ is an interval, we get that $P_{n}\left(\max J_{n}\right)=1$ 
for $a_{n}>0$ as well, when $J_{n} \neq \emptyset$. Moreover, we clearly have that $a_{[n-2 m+1, n]} \neq a_{[1,2 m]}$ for all $1 \leq m<n / 2$, thus (4) holds when $a_{n} \in B$.

(ii) If $a_{[n-2 m+1, n)}=a_{[1,2 m)}$ for some $1 \leq m<n / 2$, and $m$ is maximal with this property, then $P_{n-1}\left(\max J_{n-1}\right)=P_{2 m-1}\left(\max J_{n-1}\right)$, thus

$$
a_{2 m}+1-\left(\max J_{n-1}\right) P_{n-1}\left(\max J_{n-1}\right)=P_{2 m}\left(\max J_{n-1}\right) \in[0,1],
$$

where we have used that $J_{n-1} \subseteq J_{2 m}$ and $P_{2 m}\left(J_{2 m}\right) \subseteq[0,1]$. This gives $a_{2 m} \in B$. If $a_{n}=a_{2 m}$, then $\max J_{n}=\max J_{n-1}$ and $P_{n}\left(\max J_{n-1}\right)=P_{2 m}\left(\max J_{n-1}\right)$, thus $P_{n}\left(\max J_{n}\right)=P_{2 m}\left(\max J_{n}\right)$ and $a_{[n-2 m+1, n]}=a_{[1,2 m]}$. By the maximality of $m$, we have that $a_{[n-2 \ell+1, n]} \neq a_{[1,2 \ell]}$ for all $m<\ell<n / 2$, thus (5]) holds.

If $a_{n} \neq a_{2 m}$, then the equation $a_{[n-2 m+1, n)}=a_{[1,2 m)}$ and (1.4) yield that $a_{n}>a_{2 m}$, thus $P_{n}\left(\max J_{n}\right)=1$ when $J_{n} \neq \emptyset$, similarly to (ii). If $a_{[1,2 \ell)}=a_{[n-2 \ell+1, n)}, 1 \leq \ell<m$, then we also have that $a_{[1,2 \ell)}=a_{[2 m-2 \ell+1,2 m)}$, thus $a_{2 \ell} \leq a_{2 m}<a_{n}$. This implies that $a_{[n-2 \ell+1, n]} \neq a_{[1,2 \ell]}$ for all $1 \leq \ell<n / 2$, thus (44) holds when $a_{n} \in B$.

(iii) If $a_{[1, n-2 m)}=u_{[1, n-2 m)}$ or $a_{[n-2 m, n)} \neq a_{[1,2 m]}$ for all $1 \leq m \leq n / 2-1$, then we have that $P_{n-1}\left(\min J_{n-1}\right)=1$, thus

$$
a_{1}+1-\left(\min J_{n-1}\right) P_{n-1}\left(\min J_{n-1}\right)=P_{1}\left(\min J_{n-1}\right) \in[0,1],
$$

and $a_{1} \in B$. If $a_{n}=a_{1}$, then $\min J_{n}=\min J_{n-1}$ and $P_{n}\left(\min J_{n-1}\right)=P_{1}\left(\min J_{n-1}\right)$, thus $P_{n}\left(\min J_{n}\right)=P_{1}\left(\min J_{n}\right)$, and $a_{[1, n-2 m+1]}=u_{[1, n-2 m+1]}$ or $a_{[n-2 m+2, n]} \neq a_{[1,2 m)}$ for all $2 \leq m \leq n / 2$. Therefore, (3) holds. If $a_{n}<a_{1}$, then $P_{n}\left(\min J_{n}\right)=0$ when $J_{n} \neq \emptyset, a_{[1, n-2 m+1]}=u_{[1, n-2 m+1]}$ or $a_{[n-2 m+2, n]} \neq a_{[1,2 m)}$ for all $1 \leq m \leq n / 2$, thus (2) holds when $a_{n} \in B$.

(iv) If $a_{[1, n-2 m)} \neq u_{[1, n-2 m)}$ and $a_{[n-2 m, n)}=a_{[1,2 m]}$ for some $1 \leq m \leq n / 2-1$, and $m$ is maximal with this property, then $P_{n-1}\left(\min J_{n-1}\right)=P_{2 m}\left(\min J_{n-1}\right)$, thus

$$
a_{2 m+1}+1-\left(\min J_{n-1}\right) P_{n-1}\left(\min J_{n-1}\right)=P_{2 m+1}\left(\min J_{n-1}\right) \in[0,1],
$$

hence $a_{2 m+1} \in B$. If $a_{n}=a_{2 m+1}$, then $\min J_{n}=\min J_{n-1}$ and $P_{n}\left(\min J_{n-1}\right)=$ $P_{2 m+1}\left(\min J_{n-1}\right)$, thus $P_{n}\left(\min J_{n}\right)=P_{2 m+1}\left(\min J_{n}\right)$, and $a_{[n-2 m, n]}=a_{[1,2 m+1]}$. The maximality of $m$ yields that $a_{[1, n-2 \ell+1]}=u_{[1, n-2 \ell+1]}$ or $a_{[n-2 \ell+2, n]} \neq a_{[1,2 \ell)}$ for all $m+$ $1<\ell \leq n / 2$, thus (3) holds. If $a_{n} \neq a_{2 m+1}$, then $a_{n}<a_{2 m+1}$ by (1.4). If moreover $a_{[1,2 \ell-2]}=a_{[n-2 \ell+2, n)}, 1 \leq \ell \leq m$, then we have that $a_{[1,2 \ell-2]}=a_{[2 m-2 \ell+3,2 m]}$, thus $a_{2 \ell-1} \geq a_{2 m+1}>a_{n}$. Then we get that $P_{n}\left(\min J_{n}\right)=0$ when $J_{n} \neq \emptyset, a_{[1, n-2 \ell+1]}=$ $u_{[1, n-2 \ell+1]}$ and $a_{[n-2 \ell+2, n]} \neq a_{[1,2 \ell)}$ for all $1 \leq \ell \leq n / 2$, thus (2) holds when $a_{n} \in B$.

Since $x \mapsto x P_{n-1}(x)$ is continuous and $J_{n-1}$ is an interval, the set $B$ is an interval of integers. The paragraphs (ii) and (iii) show that $a_{n}$ is not smaller than the smallest element of $B$, (iii) and (iv) show that $a_{n}$ is not larger than the largest element of $B$, thus $a_{n} \in B$. We have therefore proved that $J_{n} \neq \emptyset$ and (2)-(5) hold, when $a_{[1, n)} \neq u_{[1, n)}$ and $n$ is even. For odd $n$, the proof runs along the same lines and is left to the reader.

If $a_{[1, n)}=u_{[1, n)}$, then $\inf J_{n-1}=1$. From [LS, Proposition 3.5], we know that $u_{n} \in B$, that inf $J_{n}=1$ when $a_{n}=u_{n}$, and that $\min J_{n}>1$ when $u_{n} \neq a_{n} \in B$. Let first $n$ be even, thus $a_{n} \leq u_{n}$ by (1.5). If $a_{[n-2 m+1, n)} \neq a_{[1,2 m)}$ for all $1 \leq m<n / 2$, then we obtain as in (ii) that $0 \in B$, thus $a_{n} \in B$, and (41) holds. If $a_{[n-2 m+1, n)}=a_{[1,2 m)}$ for some $1 \leq m<n / 2$, and 
$m$ is maximal with this property, then (iii) yields that $a_{2 m} \in B$ and $a_{2 m} \leq a_{n}$, thus $a_{n} \in B$. If $a_{n}=a_{2 m}$, then (5) holds; if $a_{n}>a_{2 m}$, then (4) holds. Moreover, if $a_{n}<u_{n}$, then we get that $P_{n}\left(\min J_{n}\right)=0$, thus (2) holds. Again, if $n$ is odd, then similar arguments apply. Hence, we have proved that $J_{n} \neq \emptyset$ and (2)-(5) hold for the case that $a_{[1, n)}=u_{[1, n)}$ too.

If $J_{n}$ is not an interval, then the continuity of $x \mapsto x P_{n-1}(x)$ on the interval $J_{n-1}$ implies that $P_{n}$ meets the lower bound 0 or the upper bound 1 at least twice within $J_{n}$. Therefore, suppose that $P_{n}(\beta)=P_{n}\left(\beta^{\prime}\right) \in\{0,1\}$ for $\beta, \beta^{\prime} \in J_{n}$. If $P_{j}(\beta) \in(0,1]$ and $P_{j}\left(\beta^{\prime}\right) \in(0,1]$ for all $1 \leq j<n$, then the $(-\beta)$-expansion of $\frac{-\beta}{\beta+1}$ and the $\left(-\beta^{\prime}\right)$-expansion of $\frac{-\beta^{\prime}}{\beta^{\prime}+1}$ are both $\overline{a_{[1, n]}}$ (if $P_{n}(\beta)=1$ ) or $\overline{a_{[1, n)}\left(a_{n}+1\right)}$ (if $P_{n}(\beta)=0$ ), thus $\beta=\beta^{\prime}$ by Theorem 3 .

Suppose in the following that $P_{j}\left(\beta^{\prime}\right)=0$ for some $1 \leq j<n$, and let $\ell \geq 1$ be minimal such that $P_{\ell}\left(\beta^{\prime}\right) \in\{0,1\}$. If $P_{\ell}\left(\beta^{\prime}\right)=0$, then $a_{\ell+1}=0$ and $P_{\ell+1}\left(\beta^{\prime}\right)=1$, hence $a_{[1, n]}$ is a concatenation of blocks $a_{[1, \ell]} 0$ and $a_{[1, \ell)}\left(a_{\ell}+1\right)$, except possibly for the last block, which is $a_{[1, \ell]}$ when $P_{n}\left(\beta^{\prime}\right)=0$. If $P_{\ell}\left(\beta^{\prime}\right)=1$, then $a_{[1, n]}$ is a concatenation of blocks $a_{[1, \ell]}$ and $a_{[1, \ell)}\left(a_{\ell}-1\right) 0$, ending with $a_{[1, \ell)}\left(a_{\ell}-1\right)$ when $P_{n}\left(\beta^{\prime}\right)=0$. We obtain that

$$
P_{n}(x)=P_{n}\left(\beta^{\prime}\right)+\left(P_{\ell}(x)-P_{\ell}\left(\beta^{\prime}\right)\right) Q(x)
$$

for some polynomial $Q(x)=\sum_{j=0}^{n-\ell} q_{j}(-x)^{j}$ with coefficients $q_{j} \in\{0,1\}$, and $q_{j-1}=q_{j-2}=$ $\cdots=q_{j-\ell+1}=0$ whenever $q_{j}=1$. If $P_{\ell}(\beta)=P_{\ell}\left(\beta^{\prime}\right)$, then the induction hypotheses yield that $\beta=\beta^{\prime}$. If $P_{\ell}(\beta) \neq P_{\ell}\left(\beta^{\prime}\right)$, then $Q(\beta)=0$, which implies that $1<\frac{1}{\beta^{\ell+1}}+\frac{1}{\beta^{2 \ell+1}}+\cdots=$ $\frac{1}{\beta^{\ell+1}-\beta}$ when $\ell$ is even, $1<\frac{1}{\beta^{\ell}}+\frac{1}{\beta^{2 \ell+1}}+\cdots=\frac{\beta}{\beta^{\ell+1}-1}$ when $\ell$ is odd, i.e., $\beta^{\ell+1}<\beta+1$.

To exclude the latter case, suppose that $P_{n}(\beta)=P_{n}\left(\beta^{\prime}\right) \in\{0,1\}$ for $\beta, \beta^{\prime} \in J_{n}, \beta \neq \beta^{\prime}$, and that $\beta^{\ell+1}<\beta+1$ for the minimal $\ell \geq 1$ such that $P_{\ell}\left(\beta^{\prime}\right) \in\{0,1\}$. Set $g_{k}=\left\lfloor 2^{k+1} / 3\right\rfloor$, and let, for $k \geq 1, \gamma_{k}$ and $\eta_{k}$ be the real numbers greater than 1 satisfying $\gamma_{k}^{g_{k}+1}=\gamma_{k}+1$, $\eta_{k}^{g_{k}+1}=\eta_{k}^{g_{k-1}+1}+1$ when $k$ is even, $\eta_{k}^{g_{k}}=\eta_{k}^{g_{k-1}}+1$ when $k$ is odd, as in [LS]. For the positive integer $m$ satisfying $g_{m} \leq \ell<g_{m+1}$, we have that $\beta<\gamma_{m}<\eta_{m}$. By Proposition 3.5 in [LS] and its proof, $\beta<\eta_{m}$ implies that the $(-\beta)$-expansion of $\frac{-\beta}{\beta+1}$ starts with $\varphi^{m}(1)$ and that $\widetilde{T}_{-\beta}^{j}(1) \notin\{0,1\}$ for all $1 \leq j \leq\left|\varphi^{m}(1)\right|=g_{m+1}+\frac{1-(-1)^{m}}{2}$, where $|w|$ denotes the length of the word $w$. Since $\beta \in J_{n}$ and $P_{n}(\beta) \in\{0,1\}$, we obtain that $a_{1} a_{2} \cdots$ starts with $\varphi^{m}(1)$ and that $n>\left|\varphi^{m}(1)\right|$. By equation (3.2) in [LS], we have that $P_{2^{m}}(x)>1$ for all $x>\eta_{m}$ (note that $\left.2^{m}=\left|\varphi^{m-1}(10)\right|<\left|\varphi^{m}(1)\right|\right)$, thus $J_{2^{m}}=\left(1, \eta_{m}\right]$, and $\ell<g_{m+1}$ yields that $\beta^{\prime}=\eta_{m}, \ell=2^{m}$. As $\beta$ and $\beta^{\prime}$ are in the interval $J_{n-1}$, we also have that $\gamma_{m} \in J_{n-1}$. The $\left(-\gamma_{m}\right)$-expansion of $\frac{-\gamma_{m}}{\gamma_{m}+1}$ is $\varphi^{m-1}(1) \overline{\varphi^{m-1}(0)}$ by [LS, Theorem 2.5]. Since $n \geq 2 \ell$ by the above block decomposition of $a_{[1, n]}$, we obtain that $a_{1} a_{2} \cdots$ starts with $\varphi^{m-1}(1000)$ if $m \geq 2$, and with 100 if $m=1$. In case $m=1$, we get that $P_{3}(2) \notin J_{3}$, contradicting that $2=\eta_{1}=\beta^{\prime} \in J_{n}$. For $m \geq 2$, we have that $P_{\left|\varphi^{m-1}(1000)\right|}\left(\eta_{m}\right)>P_{\left|\varphi^{m-1}(10)\right|}\left(\eta_{m}\right)=1$ because $P_{\left|\varphi^{m-1}(100)\right|}\left(\eta_{m}\right)=P_{\left|\varphi^{m}(1)\right|}\left(\eta_{m}\right)<P_{\left|\varphi^{m-1}(1)\right|}\left(\eta_{m}\right)$ by equation (3.4) in [LS] and, using the notation of [LS], the function $f_{\gamma_{m}, \varphi^{m-1}(0)}$ is order-reversing. Again, this contradicts that $\eta_{m}=\beta^{\prime} \in J_{n}$. Therefore, we have shown that $\beta=\beta^{\prime}$ whenever $P_{n}(\beta)=P_{n}\left(\beta^{\prime}\right) \in\{0,1\}$, $\beta, \beta^{\prime} \in J_{n}$. Hence, $J_{n}$ is an interval, and (11)-(5) hold for all $n \geq 1$.

As the $J_{n}$ form a sequence of nested non-empty intervals that are compact for sufficiently large $n$, we have that $\bigcap_{n \geq 1} J_{n} \neq \emptyset$, thus there exists some $\beta>1$ satisfying (1.9), which is 
equivalent to (1.6). To show that $\beta$ is unique, suppose that $\bigcap_{n \geq 1} J_{n}$ is not a single point. Then $\bigcap_{n \geq 1} J_{n}$ is an interval of positive length, thus there exist $\beta, \beta^{\prime} \in \bigcap_{n \geq 1} J_{n}, \beta \neq \beta^{\prime}$, such that $P_{n}(\beta) \in(0,1]$ and $P_{n}\left(\beta^{\prime}\right) \in(0,1]$ for all $n \geq 1$. This means that $a_{1} a_{2} \cdots$ is both the $(-\beta)$-expansion of $\frac{-\beta}{\beta+1}$ and the $\left(-\beta^{\prime}\right)$-expansion of $\frac{-\beta^{\prime}}{\beta^{\prime}+1}$, which contradicts that $\beta \neq \beta^{\prime}$ by Theorem 3 . This concludes the proof of Theorem 1,

Remark 1. Some parts of the proofs of Theorems 1 and 3 can be simplified when one is only interested in $\beta>1$ not too close to 1 . Since $P_{n}(x)=a_{n}+1-x P_{n-1}(x)$ for $n \geq 2$, and $P_{1}^{\prime}(x)=-1$, the derivative of $P_{n}(x)$ is

$$
P_{n}^{\prime}(x)=(-1)\left(P_{n-1}(x)+x P_{n-1}^{\prime}(x)\right)=\cdots=(-1)^{n} x^{n-1}\left(1+\sum_{j=1}^{n-1} \frac{P_{j}(x)}{(-x)^{j}}\right) .
$$

If $x \in J_{n-1}$, then $1+\sum_{j=1}^{n-1} \frac{P_{j}(x)}{(-x)^{j}}>1-\frac{1}{x}-\frac{1}{x^{3}}-\cdots=\frac{x^{2}-x-1}{x^{2}-1}$. If moreover $x \geq(1+\sqrt{5}) / 2$, then we get that $(-1)^{n} P_{n}^{\prime}(x)>0$, hence $P_{n}$ is a strictly increasing (decreasing) function on $J_{n-1} \cap[(1+\sqrt{5}) / 2, \infty)$ when $n$ is even (odd). Moreover, $\lim _{n \rightarrow \infty}\left|P_{n}^{\prime}(x)\right|=\infty$ if $x \geq$ $(1+\sqrt{5}) / 2$ and $x \in J_{n}$ for all $n \geq 1$.

However, it is not true that $P_{n}$ is always increasing (decreasing) on $J_{n-1}$ when $n$ is even (odd). For instance, if $a_{1} a_{2} \cdots$ starts with 1001, then $P_{4}(x)=x^{4}-2 x^{3}+x^{2}-x+2$ and $J_{3}=(1, \beta]$ with $\beta^{3}=2 \beta^{2}-\beta+1(\beta \approx 1.755)$. The function $P_{4}$ decreases on $\left(1, \beta^{\prime}\right]$, with $\beta^{\prime} \approx 1.261$, and increases on $\left[\beta^{\prime}, \infty\right)$. Note that this is a major flaw in the proof of Theorem 28 of [Gór07] (besides the fact that the statement is incorrect, as explained in the Introduction). This lack of monotonicity is what makes Theorems 1 and 3 more difficult to prove than the corresponding statements for $\beta$-expansions.

\section{Proof of Theorem 2}

Let $a_{1} a_{2} \cdots$ be a sequence of non-negative integers satisfying (1.4) and (1.5). We have already seen in the Introduction that these conditions are necessary to be the $(-\beta)$-expansion of $\frac{-\beta}{\beta+1}$ for some $\beta>1$. Moreover, $\beta$ can only be the number given by Theorem 11. Then $a_{1} a_{2} \cdots$ is the $(-\beta)$-expansion of $\frac{-\beta}{\beta+1}$ if and only if $\sum_{j=1}^{\infty} \frac{a_{k+j}}{(-\beta)^{j}} \neq \frac{1}{\beta+1}$ for all $k \geq 1$.

Suppose first that $\sum_{j=1}^{\infty} \frac{a_{k+j}}{(-\beta)^{j}}=\frac{1}{\beta+1}$ for some $k \geq 1$, and let $\ell \geq 1$ be minimal such that $\sum_{j=1}^{\infty} \frac{a_{\ell+j}}{(-\beta)^{j}} \in\left\{\frac{-\beta}{\beta+1}, \frac{1}{\beta+1}\right\}$. If $\sum_{j=1}^{\infty} \frac{a_{\ell+j}}{(-\beta)^{j}}=\frac{-\beta}{\beta+1}$, then the $(-\beta)$-expansion of $\frac{-\beta}{\beta+1}$ is $\overline{a_{[1, \ell]}}$. Then $a_{1} a_{2} \cdots$ is composed of blocks $a_{[1, \ell]}$ and $a_{[1, \ell)}\left(a_{\ell}-1\right) 0$. Since $\sum_{j=1}^{\infty} \frac{a_{k+j}}{(-\beta)^{j}}=\frac{1}{\beta+1}$ for some $k \geq 1$, we have at least one block $a_{[1, \ell)}\left(a_{\ell}-1\right) 0$, i.e., $a_{1} a_{2} \cdots \in\left\{a_{[1, \ell]}, a_{[1, \ell)}\left(a_{\ell}-1\right) 0\right\}^{\omega} \backslash$ $\left\{\overline{a_{[1, \ell]}}\right\}$. As $\overline{a_{[1, \ell]}}$ is the $(-\beta)$-expansion of $\frac{-\beta}{\beta+1}$, we have that $\overline{a_{[1, \ell]}}>$ alt $u_{1} u_{2} \cdots$, thus (1.7) does not hold. If $\sum_{j=1}^{\infty} \frac{a_{\ell+j}}{(-\beta)^{j}}=\frac{1}{\beta+1}$, then the $(-\beta)$-expansion of $\frac{-\beta}{\beta+1}$ is $\overline{a_{[1, \ell)}\left(a_{\ell}+1\right)}, a_{1} a_{2} \ldots$ is composed of blocks $a_{[1, \ell]} 0$ and $a_{[1, \ell)}\left(a_{\ell}+1\right)$, and we have that $\overline{a_{[1, \ell)}\left(a_{\ell}+1\right)}>_{\text {alt }} u_{1} u_{2} \cdots$, thus (1.8) does not hold. Therefore, (1.4), (1.5), (1.7), and (1.8) imply that $a_{1} a_{2} \cdots$ is the $(-\beta)$-expansion of $\frac{-\beta}{\beta+1}$ for some (unique) $\beta>1$.

Suppose now that (1.7) does not hold, i.e., $a_{1} a_{2} \cdots \in\left\{a_{[1, k]}, a_{[1, k)}\left(a_{k}-1\right) 0\right\}^{\omega} \backslash\left\{\overline{a_{[1, k]}}\right\}$ for some $k \geq 1$ with $\overline{a_{[1, k]}}>$ alt $u_{1} u_{2} \cdots$. We show that the sequence $\overline{a_{[1, k]}}$ satisfies (1.4). 
Suppose on the contrary that $a_{[j, k]} \overline{a_{[1, k]}}>_{\text {alt }} \overline{a_{[1, k]}}$ for some $2 \leq j \leq k$. This implies that $a_{[j, k]} a_{[1, j)}>_{\text {alt }} a_{[1, k]}$. Since $a_{[k+1,2 k)}=a_{[1, k)}$, we obtain that $a_{[j, j+k)}=a_{[j, k]} a_{[1, j)}>_{\text {alt }} a_{[1, k]}$, thus $a_{j} a_{j+1} \cdots>_{\text {alt }} a_{1} a_{2} \cdots$, contradicting that $a_{1} a_{2} \cdots$ satisfies (1.4). Therefore, $\overline{a_{[1, k]}}$ satisfies (1.4) and (1.5), and we can apply Theorem 1 for this sequence. Let $\beta^{\prime}>1$ be the number satisfying (11.6) for the sequence $\overline{a_{[1, k]}}$. Then $\beta^{\prime}$ also satisfies (1.6) for the original sequence $a_{1} a_{2} \cdots$, thus $\beta^{\prime}=\beta$. Therefore, $a_{1} a_{2} \cdots$ is not the $(-\beta)$-expansion of $\frac{-\beta}{\beta+1}$.

Suppose finally that (1.8) does not hold, i.e., $a_{1} a_{2} \cdots \in\left\{a_{[1, k]} 0, a_{[1, k)}\left(a_{k}+1\right)\right\}^{\omega}$ for some $k \geq 1$ with $\overline{a_{[1, k)}\left(a_{k}+1\right)}>_{\text {alt }} u_{1} u_{2} \cdots$. If $a_{1} a_{2} \cdots=\overline{a_{[1, k]} 0}$, then $\sum_{j=1}^{\infty} \frac{a_{k+j}}{(-\beta)^{j}}=\frac{1}{\beta+1}$, thus $a_{1} a_{2} \cdots$ is not the $(-\beta)$-expansion of $\frac{-\beta}{\beta+1}$. If $a_{1} a_{2} \cdots \neq \overline{a_{[1, k]} 0}$, then we show that the sequence $\overline{a_{[1, k)}\left(a_{k}+1\right)}$ satisfies (1.4). Suppose that $a_{[j, k)}\left(a_{k}+1\right) \overline{a_{[1, k)}\left(a_{k}+1\right)}>_{\text {alt }} \overline{a_{[1, k)}\left(a_{k}+1\right)}$ for some $2 \leq j \leq k$. This implies that $a_{[j, k)}\left(a_{k}+1\right) a_{[1, j)}>$ alt $a_{[1, k]}$. Since $a_{[j, k)}\left(a_{k}+1\right) a_{[1, j)}=$ $a_{[\ell, \ell+k)}$ for some $\ell \geq 2$, we have that $a_{\ell} a_{\ell+1} \cdots>_{\text {alt }} a_{1} a_{2} \cdots$, contradicting that $a_{1} a_{2} \cdots$ satisfies (1.4). As in the preceding paragraph, the number given by Theorem 1 for the sequence $\overline{a_{[1, k)}\left(a_{k}+1\right)}$ is $\beta$, thus $a_{1} a_{2} \cdots$ is not the $(-\beta)$-expansion of $\frac{-\beta}{\beta+1}$. Therefore, (1.7) and (1.8) are necessary for $a_{1} a_{2} \cdots$ to be the $(-\beta)$-expansion of $\frac{-\beta}{\beta+1}$ for some $\beta>1$.

\section{ACKNOWLEDGMENTS}

The author would like to thank Edita Pelantová for many fruitful discussions.

\section{REFERENCES}

[DMP11] D. Dombek, Z. Masáková, and E. Pelantová, Number representation using generalized (- $\beta$ )transformation, Theoret. Comput. Sci. 412 (2011), no. 48, 6653-6665.

[Fal08] B. Faller, Contribution to the ergodic theory of piecewise monotone continuous maps, Ph.D. thesis, École Polytechnique Fédérale de Lausanne, 2008.

[FL11] C. Frougny and A. C. Lai, Negative bases and automata, Discrete Math. Theor. Comput. Sci. 13 (2011), no. 1, 75-94.

[Gór07] P. Góra, Invariant densities for generalized $\beta$-maps, Ergodic Theory Dynam. Systems 27 (2007), no. $5,1583-1598$.

[IS09] S. Ito and T. Sadahiro, Beta-expansions with negative bases, Integers 9 (2009), A22, 239-259.

[LS] L. Liao and W. Steiner, Dynamical properties of the negative beta-transformation, Ergodic Theory Dynam. Systems, to appear.

[Par60] W. Parry, On the $\beta$-expansions of real numbers, Acta Math. Acad. Sci. Hungar. 11 (1960), 401-416.

[Rén57] A. Rényi, Representations for real numbers and their ergodic properties, Acta Math. Acad. Sci. Hungar. 8 (1957), 477-493.

Liafa, CNRS UMr 7089, Université Paris Diderot - Paris 7, Case 7014, 75205 Paris CEDEX 13, FRANCE

E-mail address: steiner@liafa.jussieu.fr 